\title{
Desenvolvimento de competências para Nutrição no contexto de Sistemas Alimentares Sustentáveis
}

\author{
Competency building in Nutrition in the context of Sustainable \\ Food Systems
}

Michelle Cristine Medeiros Jacob (https://orcid.org/0000-0002-4881-7285) ${ }^{1}$

Fábio Resende de Araújo (https://orcid.org/0000-0001-8268-0078) ${ }^{2}$
${ }^{1}$ Departamento de Nutrição, Universidade Federal do Rio Grande do Norte (UFRN) Av. Senador Salgado Filho 3000, Lagoa Nova. 59078970 Natal RN Brasil. michellejacob@ufrn.edu.br ${ }^{2}$ Departamento de Administração Pública e Gestão Social, UFRN. Natal RN Brasil.

\begin{abstract}
The current discussion on the impacts of food systems on human and environmental health highlights the importance of training professionals who can work on the development of the future agenda that can incorporate the complexities of nutrition into policies, research, and the rendering of services relevant to the community. This paper presents the conclusions reached at a Brazilian event in 2018 that brought together specialists on the subject, namely students and qualified specialists in nutrition committed to understanding and identifying aspects of the training that limit professional performance. Necessary requirements were identified for the development of the workforce in nutrition, including specific knowledge, a set of specific technical skills to deal with this scenario and, lastly, the necessary dialogue with other specialized areas of knowledge. Ten recommendations on the characteristics of the curriculum and practices of the professional category that could foster the construction of the necessary skills were highlighted. The recommendations may contribute to successful training actions in nutrition in the light of the growing need for alignment with the current problems of Food and Nutrition Security and full attainment of the Sustainable Development Objectives.

Key words Food and nutrition security, Competency-based education, Human resources in nutrition
\end{abstract}

Resumo A atual discussão sobre os impactos dos sistemas alimentares na saúde humana e ambiental destaca o relevo da formação de profissionais que possam atuar na elaboração de agenda futura que comporte as complexidades da Nutrição em politicas, pesquisas e prestação de serviços relevantes para a comunidade. Neste artigo são apresentadas as conclusões de um evento nacional que reuniu em 2018 especialistas no tema, estudantes e profissionais de Nutrição, empenhados em compreender e sumarizar aspectos da formação que limitam a atuação profissional. Foram identificados requisitos necessários ao desenvolvimento da força de trabalho em Nutrição, incluindo conhecimentos relativos ao tema, conjunto de habilidades técnicas especificas para fazer frente a esse cenário $e$, por fim, o diálogo necessário com outras disciplinas e áreas de conhecimento. Foram destacadas 10 recomendações relativas à característica do currículo e práticas da categoria profissional que poderão fomentar a construção das competências necessárias. As recomendações podem contribuir para a realização de ações formativas em Nutrição face à crescente necessidade de alinhamento com os problemas atuais de Segurança Alimentar e Nutricional e boa consecução dos Objetivos de Desenvolvimento Sustentável.

Palavras-chave Segurança alimentar e nutricional, Educação baseada em competências, Recursos humanos em nutrição 


\section{Introdução}

A crescente discussão sobre os impactos dos sistemas alimentares (SAs) na saúde humana e ambiental - tais como, os riscos ocupacionais aos quais estão expostos os trabalhadores do sistema agroalimentar mundial, a contaminação ambiental, a presença de alimentos contaminados, inseguros e adulterados, a disseminação de padrões dietéticos não saudáveis e a insegurança alimentar e nutricional - destaca o papel chave das agendas globais que interseccionam SAs, Sustentabilidade e Segurança Alimentar e Nutricional (SAN) ${ }^{1}$.

A SAN dos SAs é definida a partir de três dimensões básicas: disponibilidade, acesso e utilização eficiente dos recursos alimentares. A quarta, estabilidade, foi incluída em 2009 por ser indicadora da resiliência a curto prazo, por exemplo, no caso de variações climáticas, instabilidade política e fatores econômicos temporários ${ }^{2}$. Atualmente, estudiosos do tema, traçam um debate em torno da quinta dimensão, a sustentabilidade. Pensar políticas e programas de alimentação hoje, sem integração com essa ideia, pode ser a causa da crescente insegurança alimentar no futuro $^{3}$. Por isso, a sustentabilidade pode ser considerada como uma dimensão de longo prazo na avaliação da SAN dos SAs.

Em 2014, a Segunda Conferência Internacional de Nutrição, após significativos avanços na redução da fome e da má nutrição na década de 1990, apontou no seu plano de ação nove metas para atingir seu objetivo fundamental neste cenário: aprimorar, de forma sustentável, a nutrição através da implementação de políticas coerentes e de melhores ações coordenadas em todos os setores relevantes ${ }^{4}$.

Mais recentemente, em 2015, a Agenda 2030 para o Desenvolvimento Sustentável da Organização das Nações Unidas (ONU), sublinhou o relevo do tema da SAN em seu plano de ação. A meta dois do documento trata diretamente do tema: acabar com a fome, alcançar a SAN, melhorar a nutrição e promover a agricultura sustentável. Uma abordagem multidimensional Nutrição é proposta ao reconhecer suas relações com a agricultura sustentável, comércio justo, promoção da igualdade de gênero no campo, consumo, eficiência de produção, manejo de resíduos e redução do desperdício, mudanças climáticas, uso e privatização de recursos naturais, diversidade genética e cultural ${ }^{5}$.

No sentido de responder aos objetivos traçados até então, a ONU, por meio da Organização das Nações Unidas para a Alimentação e a Agri- cultura (do inglês Food and Agriculture Organization, FAO) e da Organização Mundial da Saúde (OMS), lançou a Década de Ação para Nutrição, compreendendo o período de 2016 a 2025. Um dos seis pilares estabelecidos é a ideia da construção de Sistemas Alimentares Sustentáveis (SAS) para dietas saudáveis ${ }^{6}$. A FAO denomina dietas sustentáveis como aquelas com baixo impacto ambiental, que contribuem para a SAN e para uma vida saudável para as gerações presentes e futuras. As dietas sustentáveis são protetivas e respeitadoras da biodiversidade e dos ecossistemas, culturalmente aceitáveis, economicamente justas e acessíveis, nutricionalmente adequadas, seguras e saudáveis, além de promoverem a otimização dos recursos naturais ${ }^{7}$.

Mais recentemente, a mesma agência sistematizou em cinco os princípios da agricultura e alimentação sustentável: (1) melhorar a eficiência na utilização dos recursos; (2) desenvolver ação direta para conservar, proteger e melhorar os recursos naturais; (3) proteger e melhorar os meios de subsistência rurais e bem-estar social; (4) elevar a resiliência de pessoas, comunidades e ecossistemas; e (5) aprimorar e tornar eficientes os mecanismos de governançå ${ }^{8}$.

No Brasil alguns passos vêm sendo dados com o fim de atender a essa agenda internacional. A Lei no $11.346 / 2006$, que cria o Sistema Nacional de Segurança Alimentar e Nutricional (SISAN), destaca nos seus artigos $3^{\circ}$ e $4^{\circ}$ a sustentabilidade como um dos pilares na garantia do Direito $\mathrm{Hu}$ mano à Alimentação Adequada (DHAA) 9 .

No Plano Nacional de Segurança Alimentar e Nutricional, alguns desafios foram traçados com o objetivo de atender a agenda internacional pela sustentabilidade para SAN: promover a produção de alimentos saudáveis e sustentáveis, a estruturação da agricultura familiar e o fortalecimento de sistemas de produção de base agroecológica, são alguns deles ${ }^{10}$.

Além disso, merecem destaque os esforços empreendidos na criação da Política Nacional de Agroecologia e Produção Orgânica, uma iniciativa do Governo Federal criada para ampliar e efetivar ações tendo como meta o desenvolvimento rural sustentável. Seu objetivo, de acordo com o Decreto 7.794/2012, é integrar, articular e adequar políticas, programas e ações indutoras da transição agroecológica e da produção orgânica, de base agroecológica, contribuindo para o desenvolvimento sustentável e a qualidade de vida da população, por meio do uso dos recursos naturais e da oferta e do consumo de alimentos saudáveis ${ }^{11}$. 
Diante deste cenário, destaca-se o papel-chave do profissional de Nutrição, como um dos atores diretamente implicados na produção de $\mathrm{SAS}^{12}$. Todavia, como muitas Ciências da Saúde, a Nutrição encontra-se fragmentada em tradições de pensamento que apresentam desafios para a abordagem dos SA. A abordagem hegemônica dessa ciência enfoca o nutriente, concedendo pouca ênfase às perguntas do como, onde e por quem alimentos são produzidos, processados e distribuídos e, ainda, se e como se dá o acesso da população a eles, bem como sobre a qualidade das dietas e seus impactos no ambiente ${ }^{13}$.

Faz-se necessário pensar na formação de profissionais que possam atuar na elaboração de uma agenda futura que comporte as complexidades da Nutrição a serem abordadas em políticas, pesquisas e prestação de serviços relevantes para a comunidade visando à abordagem de $\mathrm{SA}^{14}$. Essa ideia é destacada na vigésima recomendação da Segunda Conferência Internacional de Nutrição da FAO, quando menciona a necessidade de desenvolver recursos não apenas organizacionais, mas também humanos com conhecimento e habilidades necessárias à implementação de mudanças nos SA sensíveis à nutriçãó .

Diversos autores vêm desenvolvendo modelos que tentam sistematizar os pontos críticos da atual formação em relação às demandas trazidas pelas agendas globais. Tais estudos têm como ideia central o conceito de capacity building, ou desenvolvimento de competências, e se refere ao processo no qual indivíduos, organizações e a sociedade obtêm, fortalecem e mantêm as habilidades e os conhecimentos necessários para o desenvolvimento de seus objetivos ao longo do tempo ${ }^{14-18}$.

O propósito deste artigo é refletir sobre as possíveis lacunas encontradas na formação em Nutrição no que tange ao desenvolvimento de competências para atuar no contexto de SAS para SAN. Para isso, buscou-se estruturar as recomendações feitas durante o "II Ciclo de Debates sobre Sistemas Alimentares Sustentáveis para Segurança Alimentar e Nutricional", que abrigou o "I Encontro temático sobre Sistemas Alimentares Sustentáveis" da Rede Brasileira de Pesquisa em Soberania e Segurança Alimentar e Nutricional, em Natal/RN, no mês de junho de 2018. Esse evento, financiado pelo Conselho Nacional de Desenvolvimento Científico e Tecnológico $(\mathrm{CNPq})$, teve como propósito reunir especialistas no tema, estudantes e profissionais de Nutrição, empenhados em compreender e sumarizar aspectos da formação que limitam a atuação do profissional no contexto de SA. Destaca-se que participaram 32 profissionais de diversas áreas de atuação, dentre elas: Saúde Pública, Clínica, Alimentação Coletiva e Docência.

Seguindo a proposta de Fanzo et al. ${ }^{14}$ durante a oficina, os participantes foram convidados à discussão a partir das seguintes questões:

Quais conhecimentos são necessários para garantir a promoção de sistemas alimentares sustentáveis em minha área de atuação na Nutrição?

Com quais disciplinas deveria interagir para transpor os desafios apresentados? Como trabalhar de forma integrada com elas?

Que características o currículo, no nível de graduação e pós-graduação em Nutrição, deveria abarcar para possibilitar o desenvolvimento dessas competências?

O protocolo de pesquisa utilizado foi submetido ao Comitê de Ética em Pesquisa da UFRN e aprovado.

Ao princípio da oficina alguns conceitos-chave foram pactuados com o objetivo de facilitar a comunicação entre os participantes. Eles podem ser visualizados no Quadro 1.

O conteúdo das discussões foi sumarizado por relatores e socializado entre todos os participantes do evento em plenária. Neste artigo buscamos socializar as discussões feitas da seguinte forma: com o fim de aproximar o debate do cenário profissional nacional, (1) discutiremos algumas normativas que pautam a urgência do debate entre Nutrição e SAS e (2) pontuaremos, a seguir, as recomendações feitas no evento com base nas seguintes categorias: conhecimentos, habilidades, diálogo com outras disciplinas e características do currículo ${ }^{14}$. Além disso, foram adicionadas às recomendações algumas práticas da categoria profissional que poderiam fortalecer o currículo em direção a esta formação.

\section{Perspectivas normativas para o diálogo entre Nutrição e Sistemas Alimentares Sustentáveis no âmbito nacional}

O Conselho Nacional de Educação, em 2012, estabeleceu as Diretrizes Nacionais para Educação de Direitos Humanos ${ }^{19}$, recomendando a Educação em Direitos Humanos como transversal na construção dos Projetos Pedagógicos (PP) de todos os cursos de graduação. Explicita-se no parecer das diretrizes que entre os princípios da Educação em Direitos Humanos (EDH) está a Sustentabilidade socioambiental, versando que "a EDH, então, deve estar comprometida com o incentivo e promoção de um desenvolvimento 
Quadro 1. Definições pactuadas para discussão em grupo no II Ciclo de Debates.

\begin{tabular}{|l|l|}
\hline \multicolumn{1}{|c|}{ Conceito } & \multicolumn{1}{c|}{ Definição } \\
\hline $\begin{array}{l}\text { Sistema } \\
\text { alimentar }\end{array}$ & $\begin{array}{l}\text { Um sistema alimentar é definido como um sistema que abrange todos os elementos (ambiente, } \\
\text { pessoas, insumos, processos, infraestrutura, instituições, mercados e comércio) e atividades e } \\
\text { relacionadas à produção, processamento, distribuição e consumo de alimentos e os resultados } \\
\text { dessas atividades, incluindo os resultados sociais, econômicos e ambientais. }\end{array}$ \\
\hline $\begin{array}{l}\text { Dietas } \\
\text { sustentáveis }\end{array}$ & $\begin{array}{l}\text { Dietas sustentáveis são aquelas com baixo impacto ambiental, que contribuem para a segurança } \\
\text { alimentar e nutricional e para uma vida saudável para as gerações presentes e futuras. As dietas } \\
\text { sustentáveis são protetivas e respeitadoras da biodiversidade e dos ecossistemas, culturalmente } \\
\text { aceitáveis, acessíveis, economicamente justas e acessíveis, nutricionalmente adequadas, seguras e } \\
\text { saudáveis, além de promoverem a otimização dos recursos naturais. }\end{array}$ \\
\hline $\begin{array}{l}\text { Agricultura e e } \\
\text { alimentação } \\
\text { sustentável }\end{array}$ & $\begin{array}{l}\text { Agricultura e alimentação sustentável são aquelas que (1) melhoram a eficiência na utilização } \\
\text { dos recursos; (2) desenvolvem ação direta para conservar, proteger e melhorar os recursos } \\
\text { naturais, (3) protegem e melhorar os meios de subsistência rurais e bem-estar social, (4) elevam } \\
\text { a resiliência de pessoas, comunidades e ecossistemas, (5) aprimoram e tornar eficientes os } \\
\text { mecanismos de governança }\end{array}$ \\
\hline
\end{tabular}

Fonte $^{7,8,18}$.

sustentável que preserve a diversidade da vida e das culturas, condição para a sobrevivência da humanidade de hoje e das futuras gerações" ${ }^{20}$.

Registra-se ainda como marco na discussão sobre sustentabilidade na formação em cursos de graduação a Lei 9.795, de 27 de abril de 1999, que regulamenta quais conteúdos relacionados ao meio ambiente devem ser abordados transversalmente em componentes curriculares de todos os cursos de graduação. Nota-se que, conforme indicam esses documentos, o debate se faz necessário em todos os cursos ${ }^{21}$

Como diretriz principal na estruturação de PPs do Curso de Graduação em Nutrição, as Diretrizes Curriculares Nacionais (DCNs) estruturam competências básicas e específicas mínimas que devem ser pretendidas para a formação de nutricionistas. O texto no Art. 6, parágrafo primeiro, reitera que o desenvolvimento de competências deve ser induzido "considerando as demandas e necessidades prevalentes e prioritárias da população conforme o quadro epidemiológico do país/região" 22 . Percebe-se que a interseção entre SA, Sustentabilidade e SAN é demanda prioritária no quadro epidemiológico brasileiro e mundial e, portanto, deve ser considerado nas competências dos cursos de formação ${ }^{23}$.

As DCNs para a graduação em Nutrição, ainda no seu artigo 6, destaca a autonomia dos currículos em incluir "perfil, habilidades, competências e conteúdos, de forma a considerar as demandas e expectativas de desenvolvimento do setor saúde na região". Entende-se por meio deste dispositivo que o avanço da discussão dos SA exige contextualização local das estruturas curriculares. Além disso, cabe lembrar que a periodicidade de atualização dessas diretrizes (por exemplo, as atuais possuem 18 anos) não acompanha a denominação e a conceituação dos problemas na velocidade em que surgem no mundo.

O debate sobre as competências para a atuação dos nutricionistas nos sistemas sustentáveis é presente ainda no documento Consenso sobre habilidades e competências do nutricionista no âmbito da saúde coletiva ${ }^{24}$. Nesse documento, 4 competências profissionais são apontadas de modo explícito para as atuais estruturas dos cursos de formação: (1) Identificar e valorizar os aspectos referentes à sustentabilidade e ao consumo de alimentos e incorporá-los às práticas de Educação Alimentar e Nutricional; (2) Promoção do abastecimento dos alimentos com sustentabilidade; (3) Identificar e analisar a relação entre o comportamento, os hábitos alimentares, a cultura, a territorialidade, a sustentabilidade e a diversidade alimentar; e (4) Promoção da qualidade da água no contexto do DHAA e da sustentabilidade. Apesar de o documento ser especifico de uma área de atuação, Saúde Coletiva, as competências são pertinentes à atuação do nutricionista em outros segmentos.

Os postulados do II Ciclo de debates formalizam anseios que ainda não foram contemplados nas DCNs sobre o tema e sinalizam caminhos de modo prático e efetivo para o desenvolvimento de competências nessa formação. Objetivando ecoar nas estruturas curriculares de cursos de Nutrição e, por fim, influenciar normatizações, os debates tidos foram sintetizados em quatro eixos dispostos a seguir ${ }^{14}$. 


\section{Recomendações do II Ciclo}

O Quadro 2 apresenta uma síntese das Recomendações do II Ciclo de Debates.

\section{Conhecimentos para uma Nutrição sustentável}

O nutricionista em seu Código de Ética ${ }^{25}$ se compromete com o desenvolvimento sustentável e a preservação da biodiversidade, sendo esse um dos princípios fundamentais da atuação da categoria.

As áreas de atuação do nutricionista regulamentadas pela Resolução $600^{26}$ possuem conhecimentos comuns. Esta resolução também prevê como competência esperada na formação profissional a promoção de ações de incentivo ao desenvolvimento sustentável em todas as áreas de atuação. Em consonância com essas normativas, nos cursos de Graduação o tema SAS deveria ir além da sua abordagem teórica e conceitual, estando associado aos problemas da Nutrição no campo individual e coletivo em suas diversas áreas de atuação. Inter-relações entre o tema deveriam estar presentes nas disciplinas básicas de conteúdos gerais e naquelas de conteúdo especifico.

Naturalmente, pela especialização, as áreas de formação poderiam direcionar o tema dos SAS para a discussão de forma mais estreita com a formação específica, estabelecendo conexões com conhecimentos anteriores. Entende-se que os problemas atualmente identificados - diversos tipos de má nutrição no cenário de industrialização do sistema agroalimentar sob a égide neoliberal - não sejam superados com a fragmentação do conhecimento em Nutrição. O estudo dos SAS é composto de conhecimentos que vão desde conteúdos mais técnicos (biodisponibilidade de nutrientes, legislações sobre produção alimentar, técnicas de manejos de alimentos, métodos de elaboração culinária, classificações botânicas de vegetais, etc.) até aqueles mais de formação política e crítica perante às circunstâncias atuais.

É necessário que o nutricionista como pensador de SAS abandone a visão estreita e ingênua dos problemas ligados à sua atuação profissional e opere a partir de análises mais amplas, conforme pode ser visualizado na Figura 1: compreendendo o papel dos fatores ligados ao ambiente social na conformação das práticas alimentares dos sujeitos e não apenas como um campo de responsabilidades individuais, pautando-se em dietas e não em nutrientes isolados e, por fim, abordando o papel da biodiversidade dos sistemas como prioritária em relação à suplementação e biofortificação de cultivos ${ }^{1}$.

Esses conhecimentos deveriam ser abordados de forma teórica e também prática, aproximando os alunos de cenários reais e contribuindo para a formação de resolução de problemas sobre o tema. Metodologias ativas como Garden-based

Quadro 2. Síntese das discussões do II Ciclo de Debates.

\begin{tabular}{|l|l|}
\hline $\begin{array}{c}\text { Domínios para } \\
\text { construção de } \\
\text { competências }\end{array}$ & \multicolumn{1}{c|}{ Resumo do relato } \\
\hline Conhecimentos & $\begin{array}{l}\text { Dietas e/ou cardápios sustentáveis; Sustentabilidade; Biodiversidade; Sistema alimentar; } \\
\text { Análise sistêmica da transição nutricional; Desperdício de alimentos; Agrotóxicos; } \\
\text { Alimentação no contexto urbano; Mídia e alimentação; Comer local; Educação } \\
\text { Alimentar e Nutricional para sustentabilidade; Agroecologia; Ambientes alimentares. }\end{array}$ \\
\hline Habilidades & $\begin{array}{l}\text { Elaborar e avaliar dietas e cardápios sustentáveis; Gerir processos de compras } \\
\text { sustentáveis em âmbito privado e público; Elaborar e avaliar programas e políticas com } \\
\text { base em indicadores de sustentabilidade; Atuar em instâncias de participação social e } \\
\text { junto a movimentos sociais; Desenvolver projetos comunitários pautados pela ideia de } \\
\text { democracia alimentar }\end{array}$ \\
\hline $\begin{array}{l}\text { Diálogo com outras } \\
\text { disciplinas }\end{array}$ & $\begin{array}{l}\text { Ciências Agrárias, Educação, Ciências Sociais, Ecologia, Biologia, Gastronomia e Gestão } \\
\text { Pública. }\end{array}$ \\
\hline $\begin{array}{l}\text { Característica do } \\
\text { currículo e práticas } \\
\text { da categoria } \\
\text { profissional }\end{array}$ & $\begin{array}{l}\text { Previsão da temática em SAS no currículo; Previsão de componente específico; } \\
\text { Presença transversal do tema; Previsão de práticas no contexto comunitário; Horta } \\
\text { pedagógica como laboratório; Previsão de práticas em restaurantes sustentáveis; Perfil } \\
\text { docente multiprofissional; Interação com redes de trocas de experiências; Estímulo a } \\
\text { debate e práticas sobre o tema por meio de eventos e premiações; Indução da produção } \\
\text { acadêmica e formação de pesquisadores sobre o tema. }\end{array}$ \\
\hline
\end{tabular}

Fonte: dados do II Ciclo de Debates. 


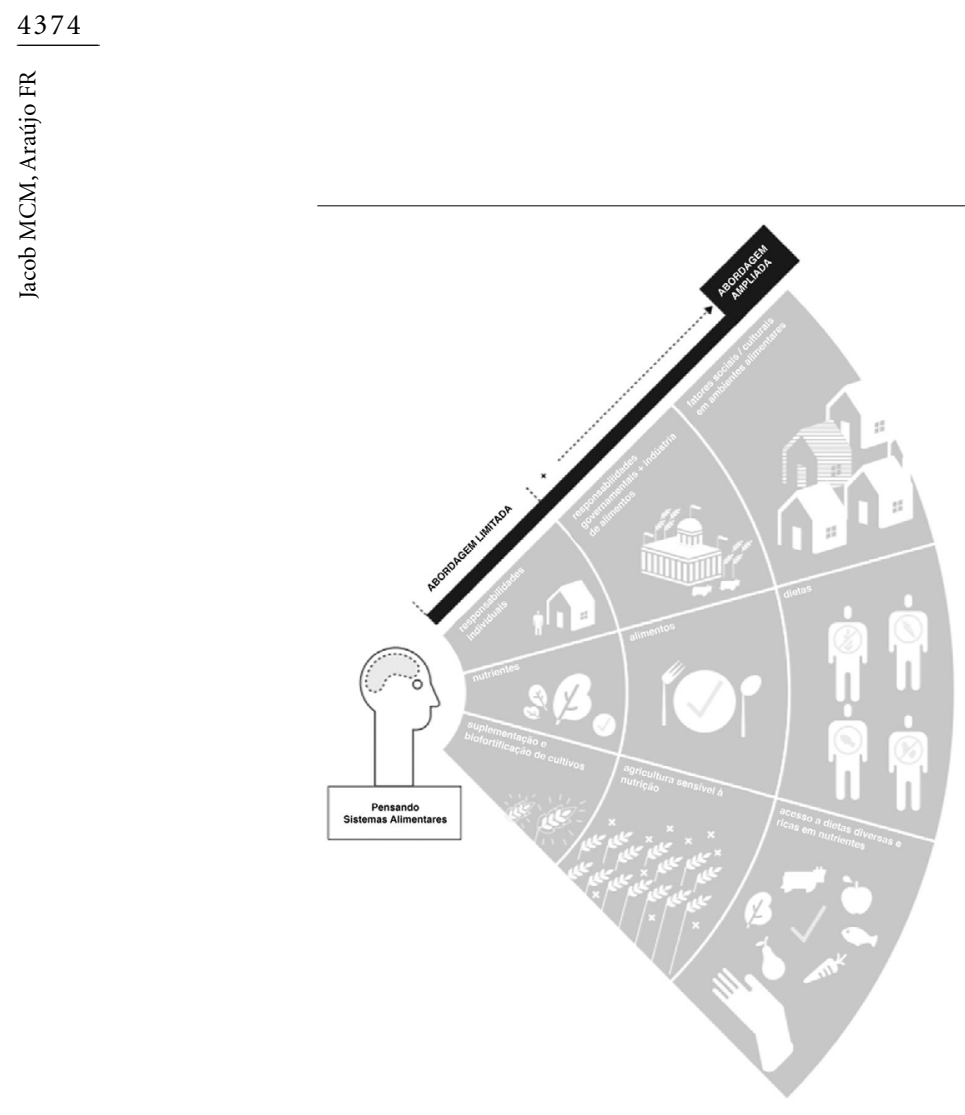

Figura 1. Ampliando o debate sobre dietas e Nutrição na formação profissional.

Fonte. Traduzido de Ipes-Food ${ }^{1}$.

learning (GBL) e Problem-based learning (PBL) são recomendadas pois são estratégias que induzem a conexão entre teoria e prática, criando situações efetivas de aprendizagem, no qual os estudantes são convidados à experimentação e colaboração para atuar sobre problemas de natureza transdisciplinar, estimulando novas vias de comunicação, aprendizagem e reflexão na ação. Em GBL ou Aprendizagem Baseada em Hortas $(\mathrm{ABH})$, por exemplo, hortas ou jardins são concebidos como instrumentos pedagógicos, onde os problemas apresentados por eles são ponto de partida para aprender ${ }^{27,28}$.

Além da necessidade de revisão dos métodos de ensino na área, faz-se necessário estimular a produção de referências sobre o tema em âmbito nacional, pois a despeito dos esforços de instituições e pesquisadores brasileiros, a literatura disponível ainda é fragmentada, sem tantos títulos específicos e aplicados. A carência de títulos em língua portuguesa ainda é um dos fatores que dificulta a capacitação dos profissionais no tema.

A exemplo do Marco de referência de Educação Alimentar e Nutricional para as políticas públicas $^{29}$, a efetiva organização do ensino e da prática em SAs para Nutrição demandaria um marco ou diretriz que organizasse os conhecimentos relativos ao campo, com a finalidade de estabelecer diretrizes formativas.

Foram citados como conhecimento vinculados ao tema, os seguintes listados:

Dietas e/ou cardápios sustentáveis

Sustentabilidade

Biodiversidade

Sistema alimentar

Análise sistêmica da transição nutricional

Desperdício de alimentos

Agrotóxicos

Alimentação no contexto urbano

Mídia e alimentação

Comer local

Educação Alimentar e Nutricional para sustentabilidade

Ambientes alimentares

\section{Habilidades para uma Nutrição sustentável}

Entre as habilidades discutidas, entendese que o nutricionista seja um profissional que deve protagonizar a discussão dos SA em todas as áreas de atuação. Este papel demanda o desenvolvimento de habilidades para atuar de forma holística, o que inclui as capacidades de liderança e de ativismo político ${ }^{30,31}$.

Entende-se que por dominar um conhecimento técnico especifico, com muitas informações disseminadas pela mídia sem critérios e em um cenário de desmonte de direitos, o nutricionista deva assumir papel de liderança no sentido de esclarecer e mobilizar os indivíduos na discussão da sustentabilidade da alimentação. As DCNs para a graduação em Nutrição, em seu artigo 4, identifica a liderança como competência básica do nutricionista, definindo-o como profissional apto a assumir posições de liderança, sempre tendo em vista o bem-estar da comunidade, e completa: "a liderança envolve compromisso, responsabilidade, empatia, habilidade para tomada de decisões, comunicação e gerenciamento de forma efetiva e eficaz." Essa habilidade, basilar nas ações de ativismo político, deve ser propulsora de mudança social.

Quanto às ações de caráter técnico que esses profissionais devem ser capacitados a realizar, enumeram-se:

Elaborar e avaliar dietas e cardápios sustentáveis;

Gerir processos de compras sustentáveis em âmbito privado e público;

Elaborar e avaliar programas e políticas com base em indicadores de sustentabilidade; 
Atuar em instâncias de participação social e junto a movimentos sociais;

Desenvolver projetos comunitários pautados pela ideia de democracia alimentar.

A Democracia Alimentar é caracterizada como um cenário em que os cidadãos têm conhecimento e se envolvem ativamente com as questões que perpassam o SA nas quais se inserem. Democracia é a forma e o procedimento idôneo para garantir que as decisões políticas sejam expressão da vontade geral, desde que se protegendo os Direitos Humanos. O regime democrático identifica quem decide, como decide e sobre o que pode ser decidido ${ }^{31-35}$.

A formação dessas competências não se restringe ao acesso a conhecimentos teóricos, mas também às atitudes e às práticas que devem ser exercitadas em disciplinas, estágios, pesquisa e extensão.

\section{Diálogo do tema da Nutrição sustentável com outras disciplinas}

Há o entendimento de que a Ciência da $\mathrm{Nu}$ trição deve estabelecer pontes e conexões de conteúdos com outras áreas disciplinares, para que assim possa dispor da base teórica sistêmica ligada à alimentação.

Áreas de conhecimento prioritárias para esse debate, podem ser citadas: Ciências Agrárias, Educação, Ciências Sociais, Ecologia, Biologia, Gastronomia e Gestão Pública. O trânsito por esses campos do conhecimento habilita o profissional para que se envolva efetivamente com os tomadores de decisão no SA.

Percebe-se que a extensão e a pesquisa são caminhos mais imediatos de aproximação destas áreas, visto que no âmbito do ensino há a compreensível morosidade decorrente dos processos de discussão e pactuação de currículos. A médio prazo a inserção de componentes específicos destas áreas podem resultar em um ensino mais comprometido com a interdisciplinaridade e, ainda, com a transdisciplinaridade na formação do nutricionista.

\section{Característica dos currículos e práticas da categoria profissional organizada para uma Nutrição sustentável}

Entende-se como urgente a inserção do tema nos cursos de graduação em Nutrição. As atuais estruturas curriculares acabam por não contribuir com a discussão, pois possuem modelos fragmentados de disciplinas, conteúdos e ativida- des. Nesse contexto, são estabelecidas frequentemente poucas e frágeis conexões entre conteúdos e, por fim, pouca compatibilidade com a solução de problemas reais. Há ainda um privilegio relativo à transmissão de conhecimentos conceituais em detrimento da formação procedimental e atitudinal. Na perspectiva de superar esses entraves, são traçadas as seguintes recomendações para o paradigma formativo proposto:

Previsão da temática em SAS no currículo. Destaca-se a previsão da temática de SAS no Projeto Pedagógico, com redação do perfil do egresso que privilegie o tema. As habilidades e as competências previstas devem estar ligadas com a capacidade de induzir e gerir SAS. Nos cursos com atuação em centro urbanos e em cidades do interior as ênfases devem se diferenciar, considerando os contextos na superação dessas realidades no acesso à alimentos adequados e saudáveis. Quanto às DCNs, recomenda-se a criação de uma competência exclusiva para promoção e gerência de SAS.

Previsão de componente específico. Pauta-se a previsão de componente de SAs relacionando sustentabilidade, SAN e Soberania nos cursos de formação em Nutrição. A relevância da previsão de um componente específico, com uso de metodologias ativas, contido na estrutura obrigatória, de abordagem teórico-prática, na estrutura curricular dos cursos, visaria a garantia da discussão do tema. Espera-se que esse seja espaço integrador de conhecimentos de outras áreas. Sugere-se como temas para a ementa, a partir das discussões realizadas, os seguintes: dimensões e indicadores de sustentabilidade; políticas de alimentação e Nutrição com ênfase na sustentabilidade; movimentos sociais implicados com a produção de sistemas alimentares sustentáveis; cardápios sustentáveis; valorização, preservação e eficiência na utilização de ingredientes locais; biodiversidade; dietas baseadas em vegetais e redução do desperdício.

Presença transversal do tema. Sugere-se que ocorra a abordagem transversal do tema, com previsão em ementa, em componentes curriculares vinculados à formação básica do nutricionista: Nutrição e dietética, Técnica dietética, Tecnologia de alimentos, Políticas públicas de alimentação e nutrição, Gestão em alimentação coletiva, Dietoterapia, Vigilância sanitária de alimentos. Além disso, essa aproximação com o tema deveria perpassar disciplinas que transcendem a formação básica: Genética, Sociologia, Economia, entre outras. Seria interessante que os conteúdos contemplassem as especificidades das áreas que se relacionam, aplicando os conhecimentos específicos aos problemas dos SAs. 
Previsão de práticas no contexto comunitário. Destaca-se o relevo do envolvimento de cenários de prática do curso (disciplinas e estágios) na comunidade, com ações voltadas para o fortalecimento da democracia alimentar. Os componentes podem trabalhar em cenários que favoreçam uma aproximação com o tema: hortas comunitárias, visita a agricultores familiares, restaurantes agroecológicos e feiras orgânicas. As atividades devem ser estruturadas com o fim de estabelecer vínculo com a comunidade, desenvolvendo atividades de impacto significativo, evitando ações pontuais e que esgotem os espaços. Nos estágios, são exemplos de cenários de atuação do nutricionista que podem estabelecer relações profícuas com o tema de SAs: Extensão rural, Vigilância sanitária, Programa Nacional da Alimentação Escolar, hospitais, Restaurantes populares, Restaurantes universitários, Banco de alimentos, entre outros. Sugere-se que essas vivências resultem em trabalhos técnicos e em ações que sejam produtivas para ambas as partes.

Horta pedagógica como laboratório. Sublinhase o relevo da previsão de hortas nos cursos de Graduação, como espaço de metodologia ativa, figurando como cenário de prática de diversos componentes de ensino, ações de pesquisa e extensão. Sugere-se que os cursos em sua estrutura física prevejam uma horta para plantio e cultivo de alimentos com bases agroecológicas. Esse espaço pode ser previsto como laboratório já no PP do curso, tal como existem laboratórios de Técnica dietética ou espaços de práticas ativas como clínicas escolas, com o fim de garantir a sustentabilidade da abordagem na formação. Esses espaços podem abarcar projetos que incentivem o conhecimento e o uso da biodiversidade local e/ ou de promoção da ação comunitária, envolvendo ações de extensão e pesquisa.

Previsão de práticas em Restaurantes Sustentáveis. Recomenda-se a previsão de práticas de ensino, pesquisa e extensão em restaurantes institucionais (universitários). O locus privilegiado desses espaços de produção e distribuição de refeições garantiria a possibilidade de experimentação sobre o tema no espaço de formação do profissional. Os seguintes temas podem ser abordados neste cenário: compras e cardápios sustentáveis, avaliação e monitoramento do uso de recursos na produção de alimentos, desperdício de alimentos, além de Educação Alimentar e Nutricional junto a manipuladores e comensais.

Perfil docente multiprofissional. Ressalta-se o relevo do perfil docente multiprofissional, incluindo este critério na contratação, visando a boa implementação do currículo. Sugere-se con- siderar na composição do quadro de docentes a presença de profissionais das áreas diversas de diálogo - Ciências Agrárias, Educação, Ciências Sociais, Ecologia, Biologia, Gastronomia e Gestão Pública - que possam realizar debates sobre o tema, assim como professores nutricionistas com formação em pós-graduação nestas áreas. Sugere-se, ainda, a previsão em editais de contratações de perfis que contemplem áreas como Humanidades e Ciências Sociais Aplicadas.

Interação com redes de troca de experiência. $\mathrm{O}$ fortalecimento e a interação com redes de intercâmbio de experiências destaca-se como ponto chave da partilha de conhecimento tático. $\mathrm{O}$ conhecimento necessário para a competência profissional é formado por dois componentes: o conhecimento explícito, formalizado e acessado por registros de escrita, e o conhecimento tácito, que contém intuições, inspirações, experiências, ideais, emoções, valores e modelos mentais. $\mathrm{O}$ conhecimento tácito não é de fácil explicação, o que torna a escrita, um meio limitado para seu compartilhamento $^{36}$. A formação de espaços digitais para relatos de experiência com fotos, vídeos, tutoriais são formas de disseminar conhecimento tácito produzido em determinados cursos, que acabam por ficar fragmentados e não conseguem ser cumulativos.

Estímulo ao debate e práticas sobre o tema por meio de eventos e premiações. Sublinha-se o relevo de eventos científicos na tarefa de pautarem seções que tratem do tema de modo a aumentar o registro de ações. De modo similar, é importante o lançamento de prêmios por entidades governamentais ou não governamentais ligadas à categoria e criação de revistas científicas (ou número especiais das já existentes) receptivas a relatos de experiência.

Indução da produção acadêmica e formação de pesquisadores sobre o tema. Destaca-se o papel das agências de fomento à pesquisa na proposição do tema em editais de financiamento. Além disso, sugere-se que linhas de pesquisa nas pós-graduação em Nutrição sejam sensíveis à abordagem da temática em dissertações e teses. Acredita-se, que medidas como essa, possam inclusive tornar os periódicos da área mais receptivos à discussão.

\section{Considerações finais}

Os membros participantes do II Ciclo de Debates, com este relato, encorajam os profissionais de Nutrição a avaliarem suas práticas pessoais e profissionais e a tomarem medidas para apoiar a sustentabilidade do SA visando a SAN. 
Não estamos alheios à criação de um nicho de mercado em torno da noção de sustentabilidade. Há poucas disciplinas científicas que são definidas por codificações tão vulneráveis aos interesses corporativos e à economia neoliberal quanto à Nutrição. Neste cenário, a capacidade política de seus profissionais, como defensores da alimentação adequada e saudável enquanto Direito Humano, precisa ser fortalecida.

O conhecimento dos profissionais de Nutrição sobre as complexas questões associadas ao SA pode avançar por meio da participação em atividades de educação continuada, extensão e pesquisa. Ressalta-se ainda a relevância da ação política em suas comunidades.

Assumimos que o exercício desenvolvido aqui requereu a necessidade de pensar o campo de atuação em alimentação e Nutrição para além do corporativismo profissional. Apesar de reconhecermos o papel de protagonismo do profissional de Nutrição nesta via que interseciona SA e Sustentabilidade SAN, é evidente a insuficiência da abordagem profissional e/ou disciplinar na resolução de questões de natureza sistêmica. Adicionamos, ainda, que se torna premente a discussão ampliada com outras categorias que partilham diretamente do problema contemporâneo ligado à alimentação e Nutrição, tais como: Engenharia de Alimentos, Tecnologia de Alimentos, Ciências Agrárias e outras.

Admitimos este como um ponto de partida em direção à reflexão do tema SAs. A tarefa de construção de competências em Nutrição sem dúvidas requer tempo, maturidade e colaboração de diversos atores. No cenário político atual brasileiro, o ativismo pela garantia da permanên- cia das instâncias de participação social faz parte dessa empreitada. Além disso, tais competências devem dialogar com as diretrizes sobre SAs e Nutrição conforme dispostas em agendas internacionais, tais como a do Committee on World Food Security (CFS), por meio do "High Level Panel of Experts on Food Security and Nutrition", ainda em elaboração. A proposta aqui apresentada é um meio de propor uma sistematização inicial e somar nossa voz junto com outros autores no âmbito nacional, em direção ao urgente debate em torno do tema.

A experiência de pensar desenvolvimento de competências em Nutrição nos ensina que a transformação de SAs não reside apenas nos novos conhecimentos necessários, mas na capacidade de utilizá-los para resolver problemas, elaborar perguntas cada vez mais complexas sobre eles, por meio do exercício de reflexão, na ação cotidiana do indivíduo consciente sobre seu papel neste cenário e, sobretudo, na assunção de que é no aprendizado de viver em comunidade que podemos realizar as transformações que tanto desejamos. Apenas a compreensão de que habitamos uma casa comum, com problemas que nos conectam e nos implicam independentemente de pátria, cor ou renda, pode unir toda humanidade na busca de um desenvolvimento sustentável e integral.

Ao ensinar competências técnicas para um nutricionista atuar visando a reforma de SAs em direção ao desenvolvimento sustentável acreditamos que o grande aprendizado deva estar relacionado a atitudes transformadoras para o bem viver em uma comunidade planetária: cooperação, diálogo, cuidado e, sobretudo, justiça.

\section{Colaboradores}

Os autores, MCM Jacob e FR Araújo, foram responsáveis pela concepção do projeto, análise e interpretação dos dados, redação do artigo ou revisão crítica relevante do conteúdo intelectual e aprovação final da versão a ser publicada.

\section{Agradecimentos}

Os autores agradecem aos participantes das oficinas desenvolvidas no Ciclo de debates, onde as ideias sintetizadas nesta publicação nasceram.

\section{Referências}

1. International Panel of Experts on Sustainable Food Systems. Unravelling the Food-Health Nexus: Addressing practices, political economy, and power relations to build healthier food systems. Brussels: Ipes Food; 2017.

2. Food and agriculture organization (FAO). Draft declaration of the world summit on food security. Rome: FAO; 2009.

3. Berry EM, Dernini S, Burlingame B, Meybeck A, Conforti P. Food security and sustainability: Can one exist without the other? Public Health Nutr 2015; 18(13):2293-2302.

4. Food and Agriculture Organization of the United Nations (FAO). Second International Conference on $\mathrm{Nu}$ trition. Rome: FAO; 2014. 
5. United Nations (UN). Transforming our world: the 2030 Agenda for Sustainable Development. New York: $\mathrm{UN} ; 2017$.

6. United Nations (UN). Decade of Action on Nutrition at the UN General Assembly (71 session). New York: $\mathrm{UN} ; 2016$.

7. Burlingame B. Sustainable Diets and biodiversity. Rome: FAO; 2012.

8. Food and agriculture organization (FAO). Building a common vision fos sustainable food and agriculture. Rome: FAO; 2014

9. Brasil. Lei $\mathrm{n}^{\circ} 11.346$, de 15 setembro 2006. Cria o Sistema Nacional de Segurança Alimentar e Nutricional - SISAN com vistas em assegurar o direito humano à alimentação adequada e dá outras providências.Diário Oficial da União 2006; 18 set.

10. Câmara Interministerial de Segurança Alimentar e Nutricional (CAISAN). Plano nacional de segurança alimentar e nutricional - 2016-2019. Brasília: CAISAN; 2016.

11. Câmara Interministerial de Agroecologia e Produção Orgânica (Ciapo). Plano Nacional de Agroecologia e Produção Orgânica - PLANAPO. Brasília: Ciapo; 2013.

12. Lang T, Barling D. Nutrition and sustainability: An emerging food policy discourse. Proc Nutr Soc 2013; 72(1):1-12.

13. Mason P, Lang T. Sustainable diets: How ecological nutrition can transform consumption and the food system. New York: Routledge; 2017.

14. Fanzo JC, Graziose MM, Kraemer K, Gillespie S, Johnston JL, de Pee S, Monterrosa E, Badham J, Bloem MW, Dangour AD, Deckelbaum R, Dobermann A, Fracassi P, Hossain M, Ingram J, Jerling JC, Jones CJ, Jap SI, Kiess L, Marshall Q, Martin K, Narayan A, Amuyunzu-Nayamongo M, Pepping F, West KP. Educating and Training a Workforce for Nutrition in a Post-2015 World. Adv Nutr An Int Rev J 2015; 6(6):639-647.

15. Shrimpton R, Hughes R, Recine E, Mason JB, Sanders D, Marks GC, Margetts B. Nutrition capacity development: A practice framework. Public Health Nutr 2014; 17(3):682-688.

16. Delisle H, Shrimpton R, Blaney S, Du Plessis L, Atwood S, Sanders D, Margetts B. Capacity-building for a strong public health nutrition workforce in lowresource countries. Bull World Health Organ 2017; 95(5):385-388.

17. Baillie E, Bjarnholt C, Gruber M, Hughes R. A capacity-building conceptual framework for public health nutrition practice. Public Health Nutr 2009; 12(8):1031-1038.

18. International Panel of Experts on Sustainable Food Systems (Ipes Food). The New Science of Sustainable Food Systems: Overcoming Barriers to Food Systems Reform. Brussels: Ipes Food; 2015.

19. Brasil. Conselho Nacional de Educação (CNE). Parecer $n^{\circ}$ 8. Estabelece Diretrizes Nacionais para Educação de Direitos Humanos. Brasília: CNE; 2012.

20. Brasil. Conselho Nacional de Educação (CNE). Resolução no 1 , de 30 de maio de 2012. Estabelece Diretrizes Nacionais para Educação de Direitos Humanos. Diário Oficial da União 2012; 31 maio.
21. Brasil. Lei 9.795, de 27 de abril de 1999. Dispõe sobre a educação ambiental, institui a Política Nacional de Educação Ambiental e dá outras providências. Diário Oficial da União 1999; 28 abr.

22. Brasil. Ministério da Educação. Resolução $n^{\circ} 5$, de 7 de novembro de 2001. Institui Diretrizes Curriculares Nacionais do Curso de Graduação em Nutrição. Diário Oficial da União 2001; 9 nov.

23. Brasil. Ministério do Desenvolvimento Social e Combate à fome (MDS). O Estado Da Segurança Alimentar E Nutricional No Brasil. Um retrato Multidimensional. Brasília: MDS; 2015.

24. Observatório de Políticas de Segurança Alimentar e Nutricional (OPSAN). Consenso sobre Habilidades e Competências do Nutricionista no Âmbito da Saúde Coletiva. São Paulo: OPSAN; 2013.

25. Conselho Federal de Nutricionistas (CFN). Resolução $n^{\circ} 599$, de 25 de fevereiro de 2018. Aprova o código de ética e de conduta do nutricionista e dá outras providências. Diário Oficial da União 2018; 25 fev.

26. Conselho Federal de Nutricionistas (CFN). Resolução $\mathrm{n}^{\circ}$ 600, de 25 de fevereiro de 2018. Dispõe sobre a definição das áreas de atuação do nutricionista e suas atribuições, indica parâmetros numéricos mínimos de referência, por área de atuação, para a efetividade dos serviços prestados à sociedade e dá outras providências. Diário Oficial da União 2018; 20 abr.

27. Food and Agriculture Organization of the United Nations (FAO). Revisiting garden-based learning in basic education. Rome: FAO; 2004.

28. Gaylie V. The Learning Garden: Ecology, Teaching, and Transformation. New York: Peter Lang; 2009.

29. Brasil. Ministério do Desenvolvimento Social e Combate à fome (MDS). Marco de Referência de Educação Alimentar e Nutricional para Políticas Públicas. Brasília: MDS; 2012.

30. Lawrence MA, Friel S, Wingrove K, James SW, Candy S. Formulating policy activities to promote healthy and sustainable diets. Public Health Nutr 2015; 18(13):2333-2340.

31. Lang T. Food control or food democracy? Re-engaging nutrition with society and the environment. $\mathrm{Pu}$ blic Health Nutr 2005; 8(6A):730-737.

32. Norberto B. O futuro da democracia: uma defesa das regras do jogo. Rio de Janeiro: Paz e Terra; 2015.

33. Ferrajoli L. Principia iuris: teoria del diritto e della democrazia. Storia e società. Bari: Editori Laterza; 2007.

34. Cademartori D, Menezes Neto E. Power, mass media and public sphere in the constitutional democracy. Seq Estud juridicos e Polit 2013; 66:187-212.

35. Hassanein N. Practicing food democracy: A pragmatic politics of transformation. J Rural Stud 2003; 19(1):77-86.

36. Takeuchi Hirotaka NI. Gestão do conhecimento. São Paulo: Bookman; 2008.

Artigo apresentado em 18/11/2018

Aprovado em 15/04/2019

Versão final apresentada em 17/04/2019 